Draft Version June 13, 2016

Preprint typeset using $\mathrm{L}^{\mathrm{A}} \mathrm{T} \mathrm{E}$ style emulateapj v. 08/22/09

\title{
CONSTRAINTS ON MACHO DARK MATTER FROM COMPACT STELLAR SYSTEMS IN ULTRA-FAINT DWARF GALAXIES
}

\author{
Timothy D. BRAndT ${ }^{1,2}$ \\ Draft version June 13, 2016
}

\begin{abstract}
I show that a recently discovered star cluster near the center of the ultra-faint dwarf galaxy Eridanus II provides strong constraints on massive compact halo objects (MACHOs) of $\gtrsim 5 M_{\odot}$ as the main component of dark matter. MACHO dark matter will dynamically heat the cluster, driving it to larger sizes and higher velocity dispersions until it dissolves into its host galaxy. The stars in compact ultra-faint dwarf galaxies themselves will be subject to the same dynamical heating; the survival of at least ten such galaxies places independent limits on MACHO dark matter of masses $\gtrsim 10 M_{\odot}$. Both Eri II's cluster and the compact ultra-faint dwarfs are characterized by stellar masses of just a few thousand $M_{\odot}$ and half-light radii of $13 \mathrm{pc}$ (for the cluster) and $\sim 30 \mathrm{pc}$ (for the ultra-faint dwarfs). These systems close the $\sim 20-100 M_{\odot}$ window of allowed MACHO dark matter and combine with existing constraints from microlensing, wide binaries, and disk kinematics to rule out dark matter composed entirely of MACHOs from $\sim 10^{-7} M_{\odot}$ up to arbitrarily high masses.
\end{abstract}

Subject headings:

\section{INTRODUCTION}

Dozens of ultra-faint dwarf galaxies have recently been discovered as satellites of the Galaxy and Andromeda, and as members of the Local Group (McConnachie 2012 and references therein; Koposov et al. 2015a; Bechtol et al. 2015; Laevens et al. 2015). These satellites have luminosities as low as $\sim 1000 L_{\odot}$, and total masses inside the half-light radius that are at least 1 3 orders of magnitude larger than their stellar masses (Simon \& Geha 2007; McConnachie 2012 and references therein). Many of them host negligible amounts of gas; all are understood to be dominated by their dark matter content.

Ultra-faint dwarf galaxies are excellent places to learn about dark matter. They currently provide the best constraints on annihilating weakly interacting massive particles (WIMPs), ruling out the simplest thermal relic cross-sections for particle masses of tens of $\mathrm{GeV}$ (Ackermann et al. 2015). Dwarf galaxies have also been a source of tension with cosmological simulations: cold dark matter simulations have long overpredicted the abundance of massive satellite galaxies (Klypin et al. 1999; Moore et al. 1999). This problem may be resolved through a combination of newly discovered dwarfs and the inclusion of baryonic physics in simulations (Brooks \& Zolotov 2014), but has also been suggested as evidence for exotic forms of dark matter or modified gravity (Lovell et al. 2012).

While the evidence for dark matter's existence is overwhelming (Spergel et al. 2003; Clowe et al. 2006; Planck Collaboration et al. 2015), the identity of the dark matter particles remains mysterious. One intriguing possibility is that the dark matter consists of black holes formed in the early Universe. Such massive compact halo objects (MACHOs, Griest 1991) could be detected in the halo of our Galaxy by gravitational microlensing

\footnotetext{
${ }^{1}$ Institute for Advanced Study, Einstein Dr., Princeton, NJ

2 NASA Sagan Fellow
}

(Paczynski 1986). Microlensing surveys, however, have now ruled out MACHOs between $\sim 10^{-7}$ and $\sim 30 M_{\odot}$ as the dominant component of dark matter in our Galaxy (Alcock et al. 2001; Tisserand et al. 2007). At MACHO masses $\gtrsim 100 M_{\odot}$, the existence of fragile, wide halo binaries constrains their abundance (Chanamé \& Gould 2004; Yoo et al. 2004), though these limits rely heavily on just a few systems (Quinn et al. 2009). Quinn et al. (2009) showed that one binary used by Yoo et al. (2004) to claim constraints for MACHOs $\gtrsim 20 M_{\odot}$ is likely spurious, which removes the constraints for masses $<200 M_{\odot}$.

At MACHO masses ¿100 $M_{\odot}$, Hernández et al. (2004) showed that some dwarf galaxy cores would be dynamically relaxed, in tension with the relatively constant-density cores that have been inferred (Sánchez-Salcedo et al. 2006; Goerdt et al. 2006). MACHOs of very high mass $\left(\gtrsim 10^{7} M_{\odot}\right)$ are also ruled out by the kinematics of the Galactic disk (Lacey \& Ostriker 1985). The only constraints on a population of MACHOs between $\sim 30 M_{\odot}$ and $\sim 100 M_{\odot}$, however, currently come from limits on spectral distortions of the cosmic microwave background (CMB): black holes may have accreted during the early Universe, leaving an imprint on the CMB (Ricotti et al. 2008). However, other authors have argued that these constraints may not be definitive (Bird et al. 2016). Muñoz et al. (2016) have shown that MACHOs of these masses may also be probed by lensed fast radio bursts (FRBs).

In this paper, I derive MACHO constraints from the compact stellar distributions of ultra-faint dwarf galaxies and, in particular, from the survival of a star cluster in Eridanus II. Eridanus II was discovered as part of the Dark Energy Survey (Koposov et al. 2015a; Bechtol et al. 2015). It has an absolute magnitude of $M_{V}=-7.1$ and a half-light radius of almost $300 \mathrm{pc}$, and lies just beyond the Galaxy's virial radius at a distance of $366 \pm 17 \mathrm{kpc}$ (Crnojević et al. 2016). Eri II hosts a single star cluster of absolute magnitude $M_{V}=-3.5$ $\left(\sim 2000 L_{\odot, V}\right)$ and half-light radius $r_{h}=13 \mathrm{pc}$. The 
star cluster appears to be nearly coincident with the galaxy's center. Several other ultra-faint dwarfs have stellar masses of a few thousand $M_{\odot}$ and half-light radii of $\sim 30$ pc (Bechtol et al. 2015; Koposov et al. 2015a, b; Laevens et al. 2015); these galaxies provide independent MACHO constraints.

Eri II is one of the few dwarf galaxies with a star cluster, but it is not unique in this respect. The Fornax dwarf spheroidal galaxy has long been known to host globular clusters (Baade \& Hubble 1939; Hodge 1961). Its five known globular clusters range from $240 \mathrm{pc}$ to $1.6 \mathrm{kpc}$ in projected separation from the galaxy center, and from $\sim 4 \times 10^{4} M_{\odot}$ to $\sim 3 \times 10^{5} M_{\odot}$ in mass Mackey \& Gilmore 2003). Dynamical friction should cause these clusters to spiral in towards the center of Fornax (Tremaine 1976); their current existence outside of its core places interesting constraints on the properties of Fornax's dark halo (Sánchez-Salcedo et al. 2006; Goerdt et al. 2006; Inoue 2011; Cole et al. 2012). Other dwarf galaxies do host nuclear star clusters (Georgiev et al. 2009); these are invariably much more massive and more tightly bound than the cluster in Eri II.

In this paper I show that the star cluster in Eri II has important implications for MACHO dark matter, and that the population of compact ultra-faint dwarfs provides similar, independent limits. The paper is organized as follows. In Section 2, I apply the theory of collisional stellar systems to dynamical heating of the cluster by MACHOs. Section 3 presents the constraints on MACHO dark matter from the cluster in Eri II and from the population of compact ultra-faint dwarfs. I discuss and conclude with Section 4

\section{HEATING OF A STAR CLUSTER BY MACHOS}

A star cluster is a dynamic environment where gravitational interactions lead to the exchange of energy between stars. These interactions cause the system to dynamically relax; they may be approximated as diffusion terms using the Fokker-Planck equation (Chandrasekhar 1943). This approximation has enabled modeling of cluster density and velocity distributions (King 1966; Mevlan \& Heggie 1997 and references therein). When a range of masses is present, stellar interactions lead to mass segregation, in which the most massive bodies have the most spatially compact distribution (Spitzer 1969).

Two-body interactions tend to equalize the mean kinetic energies of different mass groups at a given location. In a system consisting of $>1 M_{\odot}$ MACHOs and stars, the stars will gain energy from the MACHOs; a compact stellar system will puff up. This can be treated as a diffusion problem, with weak scatterings gradually changing each star's velocity. The sum of the diffusion coefficients for the parallel and perpendicular components of the velocity describes the evolution of a star's kinetic energy. Assuming an isotropic Maxwellian velocity distribution for the dark matter particles and a locally uniform dark matter density, the relevant diffusion coefficient is given by

$$
D\left[(\Delta v)^{2}\right]=\frac{4 \sqrt{2} \pi G^{2} f_{\mathrm{DM}} \rho m_{a} \ln \Lambda}{\sigma}\left[\frac{\operatorname{erf}(X)}{X}\right],
$$

where $\ln \Lambda$ is the Coulomb logarithm, $m_{a}$ and $\sigma$ are the $\mathrm{MACHO}$ mass and velocity dispersion, $\rho$ is the total dark matter density, and $f_{\mathrm{DM}}$ is the fraction of dark matter in MACHOs of mass $m_{a}$ (Binney \& Tremaine 2008). The variable $X$ is the ratio of the stellar velocity to that of the MACHOs, $X \equiv v_{*} /(\sqrt{2} \sigma)$. I will assume that the stars are relatively cold compared to the dark matter, $v_{*} \lesssim \sigma$, which implies that $\operatorname{erf}(X) / X \sim 1$. This assumption is satisfied for all of the cluster and dark matter parameters used in the following section. For the Coulomb logarithm,

$$
\ln \Lambda \approx \ln \left(\frac{b_{\max }\left\langle v^{2}\right\rangle}{G\left(m+m_{a}\right)}\right) \approx \ln \left(\frac{r_{h} \sigma^{2}}{G\left(m+m_{a}\right)}\right),
$$

where $m$ and $m_{a}$ are the masses of the cluster stars and MACHOs, respectively, $\left\langle v^{2}\right\rangle \approx \sigma^{2}$ is the typical relative velocity, and $b_{\max }$ is the maximum impact parameter (Binney \& Tremaine 2008), which I take to be the halflight radius $r_{h}$. For $10 M_{\odot}$ MACHOs with $\sigma=10 \mathrm{~km} \mathrm{~s}^{-1}$ and $r_{h}=13 \mathrm{pc}, \ln \Lambda \approx 10$. As usual, the Coulomb logarithm is very insensitive to the assumed halo properties.

If dark matter is a mixture of MACHOs and low-mass particles like WIMPs, dynamical heating will compete with dynamical cooling. The cooling rate from WIMPs is given by

$D[\Delta E]=v D\left[\Delta v_{\|}\right]=-\frac{4 \pi G^{2} \rho v_{*} m_{*}\left(1-f_{\mathrm{DM}}\right) \ln \Lambda}{\sigma^{2}} G(X)$,

with $X \equiv v_{*} /(\sqrt{2} \sigma)$ as before, $m_{*}$ being the mass of a typical star, and

$$
G(X)=\frac{1}{2 X^{2}}\left[\operatorname{erf}(X)-\frac{2 X}{\sqrt{\pi}} \exp -X^{2}\right]
$$

Dynamical heating will dominate over cooling by a factor

$$
\frac{\text { heating }}{\text { cooling }} \sim \frac{m_{a} \sigma}{\sqrt{2} m_{*} v_{*}}\left(\frac{\operatorname{erf}(X)}{X G(X)}\right)\left(\frac{f_{\mathrm{DM}}}{1-f_{\mathrm{DM}}}\right),
$$

or a factor of $\sim 100 f_{\mathrm{DM}} /\left(1-f_{\mathrm{DM}}\right)$ for $10 M_{\odot}$ MACHOs. Equation (5) is always much larger than unity for the limiting $f_{\mathrm{DM}}$ derived in the following section; I neglect WIMP cooling for the rest of this paper.

Heating by MACHOs will add energy to the cluster, causing it to expand. If the cluster is embedded in a dark matter core of constant density $\rho$, its potential energy per unit mass is given by

$$
\frac{U}{M}=\text { constant }-\alpha \frac{G M_{*}}{r_{h}}+\beta G \rho r_{h}^{2},
$$

where $M_{*}$ is the cluster's stellar mass, $r_{h}$ is its projected half-mass radius, and $\alpha$ and $\beta$ are proportionality constants that depend on the mass distribution. For a cored Sérsic profile, $\alpha \sim 0.4$ and $\beta \sim 10$; I will adopt these values throughout the paper and assume them to remain constant as the cluster expands. The measured profile of the cluster in the dwarf galaxy Eridanus II is a Sérsic profile with $n \approx 0.2$ (Crnojević et al. 2016). Performing the integrals using the python package sersic (Novak et al. 2012) gives values of $\alpha \approx 0.36$ and $\beta \approx 7.2$, which would make this cluster expand slightly faster than with my fiducial $\alpha$ and $\beta$.

Using the virial theorem, $E_{\text {tot }}=-\frac{1}{2} U$, Equations (6) and (11) combine to give an implicit equation for the evo- 


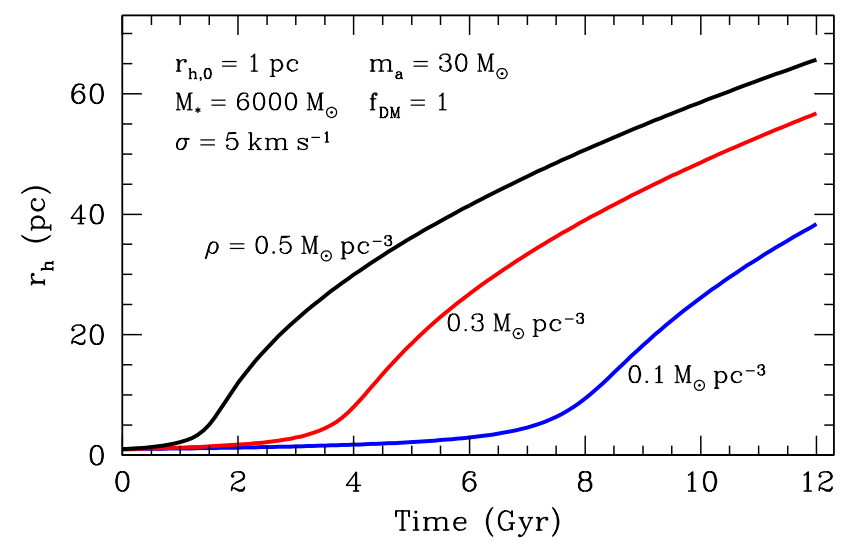

Fig. 1.- Dynamical heating of a $6000 M_{\odot}$ star cluster by $30 M_{\odot}$ MACHOs at three fiducial densities, neglecting mass loss from the cluster. The cluster expands slowly until its mean density equals that of the MACHOs, and then expands as $r_{h} \sim \sqrt{t}$.

lution of the half-light radius,

$$
\frac{d r_{h}}{d t}=\frac{4 \sqrt{2} \pi G f_{\mathrm{DM}} m_{a}}{\sigma} \ln \Lambda\left(\alpha \frac{M_{*}}{\rho r_{h}^{2}}+2 \beta r_{h}\right)^{-1} .
$$

As long as the star cluster is dark-matter dominated, Equation (7) is independent of the dark matter density. A compact stellar system will expand slowly until it becomes dominated by its dark matter content, and then expand with $r_{h} \sim \sqrt{t}$. Figure 1demonstrates this behavior for a $6000 M_{\odot}$ cluster with an initial half-light radius of $1 \mathrm{pc}$ for $30 M_{\odot}$ MACHOs at three fiducial dark matter densities, taking $\alpha=0.4$ and $\beta=10$.

Motivated by Equation (7), I define two characteristic lifetimes for a stellar system. The first is the time for it to puff up to its observed size from the $\sim 2$ pc core radius of a typical Galactic star cluster (Kharchenko et al. 2005; Harris 1996, 2010 edition). The second is the timescale to double in area (increase by a factor of $\sqrt{2}$ in linear size). In the limit of a dark-matter dominated system, these timescales are equal to each other, and to the timescale for the cluster to gain energy equal to its current kinetic energy.

\section{CONSTRAINTS FROM THE ULTRA-FAINT DWARFS}

I now combine Equation (7) with the observed survival of compact ultra-faint dwarf galaxies and of the star cluster in the core of Eridanus II to constrain MACHO dark matter. As described in the previous section, I define two characteristic lifetimes: (1) the time for the cluster to expand to its current size from the $\sim 2$ pc core of a typical Galactic cluster; and (2) the time to double its current area. By requiring these times, derived using Equation (17), to be longer than the cluster's age, I derive corresponding constraints on the abundance of MACHO dark matter.

\subsection{The Cluster in Eridanus II}

The star cluster in Eridanus II is believed to be at least $\sim 3$ Gyr old, and could be as old as $\sim 12$ Gyr (Crnojević et al. 2016). At an age of 3 Gyr, the $V$ band mass-to-light ratio for a metal-poor stellar system is $\sim 1 M_{\odot} / L_{\odot}$, while this ratio is $\sim 3 M_{\odot} / L_{\odot}$ for an old system (Maraston 2005). The cluster's observed $M_{V}=-3.5$ thus implies a stellar mass of $\sim 2000 M_{\odot}$ at an age of 3 Gyr, or a mass of $\sim 6000 M_{\odot}$ at an age of 12 Gyr. The system has an observed half-light radius $r_{h}=13$ pc. I assume the system to have resided within the core of the dark matter halo for its entire life, and derive MACHO limits by requiring the timescales for dynamical heating to be longer than the cluster's age.

Figure 2 shows the constraints for a range of plausible dark matter halo properties, with three-dimensional velocity dispersions of $5-10 \mathrm{~km} \mathrm{~s}^{-1}$ and dark matter densities of $0.02-1 M_{\odot} \mathrm{pc}^{-3}$. These values span the range of parameters characteristic of ultra-faint dwarf galaxies (Simon \& Geha 2007; McConnachie 2012 and references therein). At an age of 3 Gyr (left panel), MACHOs $\lesssim 15 M_{\odot}$ are excluded from making up all of the dark matter unless the Eri II cluster was initially compact and remains embedded in a low-density, high-dispersion halo. In this case, a cluster of the observed size is a transient phenomenon; similar objects should be rarer than compact low-mass clusters. If the cluster has spent $\sim 12 \mathrm{Gyr}$ near the center of its halo (right panel), the constraints strengthen.

The preceding discussion assumed a roughly constant dark matter density profile (a core larger than the cluster). Assuming a cuspy dark matter profile with the cluster at the dynamical center would strengthen the conclusions. Such an assumption would make the cluster dominated by dark matter at a smaller half-light radius; it would quickly begin to evolve with $r_{h} \sim \sqrt{t}$ independently of dark matter density (Equation (7D)). Further, the velocity dispersion of the dark matter particles is expected to fall toward the center of an NFW halo (Ferrer \& Hunter 2013). Lower velocity dispersions would make MACHOs even more effective at dynamical heating, improving constraints on their abundance. If, on the other hand, the cluster were slightly offset from the dynamical center of a strong dark matter cusp, it would be tidally shredded in a dynamical time.

\subsection{Constraints from Other Ultra-Faint Dwarfs}

The entire stellar population of a dwarf galaxy will also be dynamically heated by MACHOs. Many compact ultra-faint dwarf galaxies are now known, with stellar masses $\lesssim 3000 M_{\odot}$ (assuming a mass-to-light ratio $\left.M / L_{V}=3 \widetilde{M}_{\odot} / L_{\odot, V}\right)$, half-light radii $\lesssim 30 \mathrm{pc}$, and central densities $\sim 1 M_{\odot} \mathrm{pc}^{-3}$. Table11ists some basic properties of ten compact ultra-faint dwarfs (plus the star cluster in Eri II); all but three were discovered since 2015. Where measured, the ages of the stars are consistent with 10 Gyr (Bechtol et al. 2015; Laevens et al. 2015). The mean densities listed are vulnerable to different definitions of the half-light or half-mass radius, and should be treated as uncertain to at least a factor of $\sim 2$. The compact ultra-faint dwarfs constrain MACHO dark matter in the same way as the star cluster in Eri II: I use the same two heating timescales and require one or the other to be longer than 10 Gyr.

Figure 3 shows the limits on MACHO dark matter implied by a fiducial compact ultra-faint dwarf, with $r_{h}=30 \mathrm{pc}, M_{*}=3000 M_{\odot}$, and a central dark matter density $\rho=1 M_{\odot} \mathrm{pc}^{-3}$, for three-dimensional velocity 

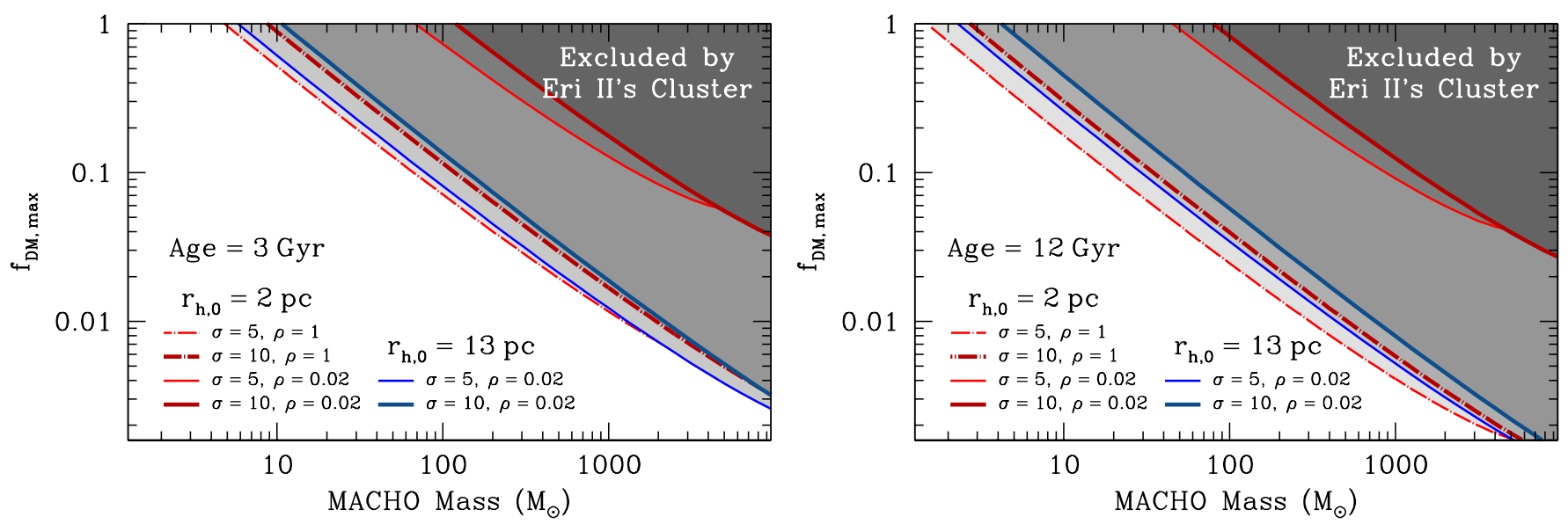

FIG. 2.- MACHO constraints from the survival of the star cluster near the core of Eridanus II, assuming a cluster age of 3 Gyr (left panel) and 12 Gyr (right panel). The units for the dark matter density $\rho$ and velocity dispersion $\sigma$, are $M_{\odot} \mathrm{pc}^{-3}$ and km s $^{-1}$, respectively. The limits come from requiring that the timescale to grow from $r_{h, 0}=2 \mathrm{pc}$ to the observed $r_{h}=13 \mathrm{pc}$ is longer than the cluster age (red lines), or from requiring that the timescale to double in area (increase by $\sqrt{2}$ in $r_{h}$ ) is longer than the cluster age (blue lines).

TABLE 1

Properties of Compact Ultra-Faint Dwarf Galaxies

\begin{tabular}{lccccr}
\hline \hline Name & $\begin{array}{c}r_{h}{ }^{\dagger} \\
\mathrm{pc}\end{array}$ & $\begin{array}{c}L_{V} \\
L_{\odot}\end{array}$ & $\begin{array}{c}\bar{\rho}_{1 / 2} \\
M_{\odot} \mathrm{pc}^{-3}\end{array}$ & $\begin{array}{c}\sigma_{*} \\
\mathrm{~km} \mathrm{~s}^{-1}\end{array}$ & Ref. $^{\dagger \dagger}$ \\
\hline Wil I & $25 \pm 6$ & 1000 & 4 & $4.3_{-1.3}^{+2.3}$ & 1,2 \\
Seg I & $29_{-5}^{+8}$ & 300 & 3 & $3.9 \pm 0.8$ & 2,3 \\
Seg II & $35 \pm 3$ & 900 & 1 & $3.4_{-1.2}^{+2.5}$ & 4 \\
Ret II & $32_{-1}^{+2} / 55_{-5}^{+5}$ & $1500^{*}$ & 2 & $3.2_{-0.5}^{+1.6}$ & $5,6,7$ \\
Hor I & $30_{-3}^{+4} / 60_{-30}^{+76}$ & $2000^{*}$ & 5 & $4.9_{-0.9}^{+2.8}$ & $5,6,7$ \\
Pic I & $29_{-4}^{+9} / 43_{-21}^{+153}$ & $2000^{*}$ & & & 5,6 \\
Pho II & $26_{-4}^{+6} / 33_{-11}^{+20}$ & $1500^{*}$ & & & 5,6 \\
Ind I & $37_{-8}^{+13} / 12_{-2}^{+2}$ & $1000^{*}$ & & & 5,6 \\
Eri III & $14_{-3}^{+13} / 11_{-5}^{+8}$ & $500^{*}$ & & & 5,6 \\
Dra II & $19_{-6}^{+8}$ & 1000 & & & 8 \\
Eri II & $13 \pm 1$ & 2000 & & & 9 \\
\hline
\end{tabular}

$\dagger$ Where two values are given, the first is from Koposov et al. (2015a) and the second from Bechtol et al. (2015).

$\dagger \dagger$ References abbreviated as: 1 (Martin et al. 2007); 2 (Martin et al. 2008); 3 (Simon et al. 2011); 4 (Belokurov et al. 2009); 5 (Bechtol et al. 2015); 6 (Koposov et al. 2015a); 7 (Koposov et al. 2015b); 8 (Laevens et al.|2015); 9 (Crnojević et al. 2016)

* Geometric means of Koposov et al. (2015a) and Bechtol et al. (2015), rounded to $500 L_{\odot}$.

Values are for the central star cluster only.

dispersions of 5 and $10 \mathrm{~km} \mathrm{~s}^{-1}$. The observed ultra-faint dwarfs lie within this range; with one-dimensional velocity dispersions between 3 and $6 \mathrm{kms}^{-1}$ (Table 11). The survival of the compact ultra-faint dwarfs listed in Table 1 rules out dark matter consisting entirely of MACHOs of mass $\gtrsim 10 M_{\odot}$.

\section{DISCUSSION AND CONCLUSIONS}

The star cluster in the core of the newly discovered dwarf galaxy Eridanus II provides strong constraints on a region of MACHO parameter space difficult to probe with either microlensing or wide Galactic binaries; the population of compact, ultra-faint dwarfs provides similar, independent limits. Figure 4 compares the constraints derived in Section 3 using conservative assump-

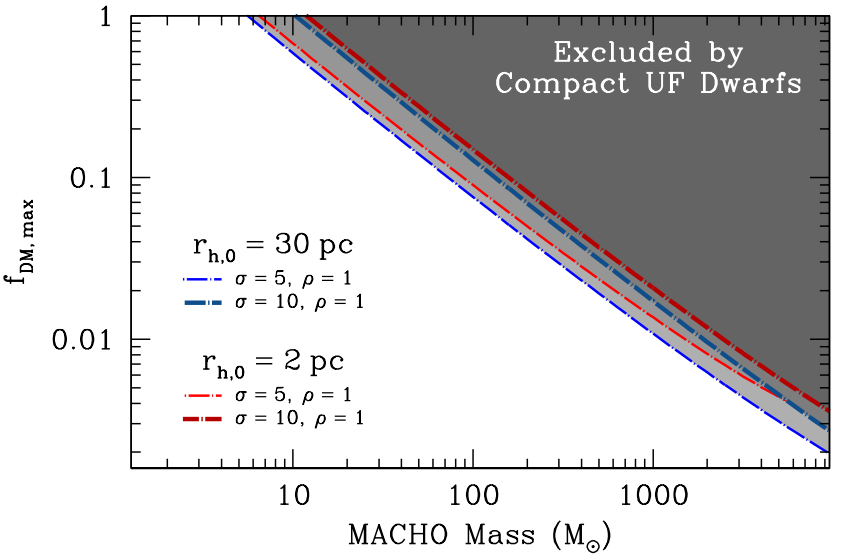

FIG. 3.- MACHO constraints from the observed sizes of compact ultra-faint dwarf galaxies, assuming a stellar mass of $3000 M_{\odot}$, a current half-light radius $r_{h}=30 \mathrm{pc}$, and an age of $10 \mathrm{Gyr}$. The units for the dark matter density $\rho$ and velocity dispersion $\sigma$, are $M \odot \mathrm{pc}^{-3}$ and $\mathrm{km} \mathrm{s}^{-1}$, respectively. The limits come from requiring that the timescale to grow from $r_{h, 0}=2 \mathrm{pc}$ to $r_{h}=30 \mathrm{pc}$ is longer than 10 Gyr (red lines), or from requiring that the timescale to double in area (increase by $\sqrt{2}$ in $r_{h}$ ) is longer than 10 Gyr (blue lines).

tions about the dark matter halos to constraints from microlensing (Alcock et al. 2001; Tisserand et al. 2007) and wide Galactic halo binaries (Quinn et al. 2009). The kinematics of the Galactic disk provide an independent limit on the abundance of very massive $\left(\gtrsim 10^{7} M_{\odot}\right)$ MACHOs (Lacey \& Ostriker 1985). For dark matter halos consistent with measured dwarf properties (Table 1), MACHO dark matter is ruled out over the entire open region of masses. If Eri II's cluster is old and was initially puffier than Galactic clusters, it provides especially strong limits.

While Eri II's cluster likely provides the best limits on MACHOs from $\sim 10 M_{\odot}$ up to thousands of $M_{\odot}$, there are ways to evade its constraints. The cluster, for example, could have recently spiraled into the center of Eri II due to dynamical friction, having spent most of its life as a compact cluster in a low-density MACHO environment. However, the inspiral timescale is inversely pro- 


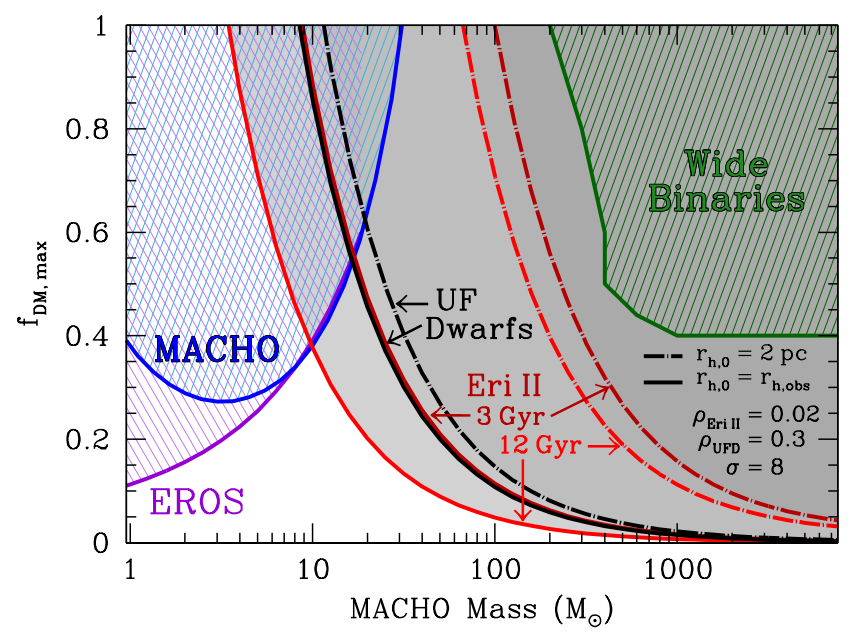

FIG. 4.- Constraints on MACHO dark matter from microlensing (blue and purple, Alcock et al. 2001; Tisserand et al. 2007) and wide Galactic binaries (green, Quinn et al. 2009), shown together with the constraints from the survival of compact ultra-faint dwarf galaxies and the star cluster in Eridanus II. I conservatively adopt a dark matter density of $0.02 M_{\odot} \mathrm{pc}^{-3}$ in Eri II and $0.3 M_{\odot} \mathrm{pc}^{-3}$ in the ultra-faint dwarfs, assume a three-dimensional velocity dispersion $\sigma=8 \mathrm{~km} \mathrm{~s}^{-1}$, and use two definitions of the heating timescale. A low-density halo and initially compact cluster weaken the constraints from Eri II. Even in this case, assuming dark matter halos to have the properties that are currently inferred, MACHO dark matter is excluded for all MACHO masses $\gtrsim 10^{-7} M_{\odot}$.

portional to the cluster mass (Binney \& Tremaine 2008), and the cluster in Eri II is 1.5-2 orders of magnitude less massive than Fornax 4 (Mackey \& Gilmore 2003), the Fornax globular cluster nearest the center of that dwarf (at $240 \mathrm{pc}$ in projected separation). This scenario therefore requires very different dark matter halos in the two galaxies or severe mass loss during Eri II's inspiral, and also luck to catch the cluster on the point of disruption. This problem of coincidence is generic to any scenario in which Eri II's cluster was initially compact. The probability of observing the system in such a transient state is significantly higher if the cluster's age is $\sim 3$ Gyr rather than $\sim 12$ Gyr.

Other possibilities to evade the constraints include an intermediate-mass black hole $\left(\gtrsim 10^{4} M_{\odot}\right)$ to provide binding energy, or a chance alignment such that the cluster only appears to reside in the center of Eri II. Both would be surprising. Such a black hole would have a mass comparable to the total stellar mass of its host galaxy. A massive black hole would also be expected to host a relaxed MACHO cluster of comparable mass, in which case it may not avoid the problem of dynamical heating at all. A chance alignment of a cluster physically located at the galaxy's half-light radius is possible; the most naïve estimate, the fraction of solid angle lying within a few $r_{h}$ in projection, gives a chance alignment probability of $\sim 1 \%$ at a physical distance of $\sim 300 \mathrm{pc}$ from the galaxy core.

While many scenarios could, in principle, account for the survival of the star cluster in Eri II, it is harder to appeal to coincidence for the entire sample of compact ultra-faint dwarfs. Assuming the measured velocity dispersions to reflect the properties of their dark matter halos, these dwarfs should have much larger half-light radii if their dark matter is all in the form of MACHOs $>10 M_{\odot}$. The strongest constraints, however, may come from the cluster in Eri II, and could be improved with better data. Precise photometry with the Hubble Space Telescope could resolve the question of whether the cluster is intermediate-age or old, while spectroscopy of cluster members and nonmembers would give another probe of Eri II's dark matter content. While future observations will determine the strength of the constraints from Eri II, existing data from Eri II and from the sample of compact ultra-faint dwarfs appear sufficient to rule out dark matter composed exclusively of MACHOs for all masses above $\sim 10^{-7} M_{\odot}$.

I thank Ben Bar-Or, Juna Kollmeier, Kris Sigurdson, and especially Scott Tremaine for helpful conversations and suggestions, and an anonymous referee for helpful comments. This work was performed under contract with the Jet Propulsion Laboratory (JPL) funded by NASA through the Sagan Fellowship Program executed by the NASA Exoplanet Science Institute.

\section{REFERENCES}

Ackermann, M., Albert, A., Anderson, B., et al. 2015, Physical Review Letters, 115, 231301

Alcock, C., Allsman, R. A., Alves, D. R., et al. 2001, ApJ, 550, L169

Baade, W., \& Hubble, E. 1939, PASP, 51, 40

Bechtol, K., Drlica-Wagner, A., Balbinot, E., et al. 2015, ApJ, 807, 50

Belokurov, V., Walker, M. G., Evans, N. W., et al. 2009, MNRAS, 397, 1748

Binney, J., \& Tremaine, S. 2008, Galactic Dynamics: Second Edition (Princeton University Press)

Bird, S., Cholis, I., Muñoz, J. B., et al. 2016, ArXiv e-prints, 1603.00464

Brooks, A. M., \& Zolotov, A. 2014, ApJ, 786, 87

Chanamé, J., \& Gould, A. 2004, ApJ, 601, 289

Chandrasekhar, S. 1943, Reviews of Modern Physics, 15, 1

Clowe, D., Bradač, M., Gonzalez, A. H., et al. 2006, ApJ, 648, L109

Cole, D. R., Dehnen, W., Read, J. I., \& Wilkinson, M. I. 2012, MNRAS, 426, 601

Crnojević, D., Sand, D. J., Zaritsky, D., et al. 2016, ArXiv e-prints, 1604.08590

Ferrer, F., \& Hunter, D. R. 2013, JCAP, 9, 005

Georgiev, I. Y., Hilker, M., Puzia, T. H., Goudfrooij, P., \& Baumgardt, H. 2009, MNRAS, 396, 1075
Goerdt, T., Moore, B., Read, J. I., Stadel, J., \& Zemp, M. 2006, MNRAS, 368, 1073

Griest, K. 1991, ApJ, 366, 412

Harris, W. E. 1996, AJ, 112, 1487

Hernández, X., Matos, T., Sussman, R. A., \& Verbin, Y. 2004 PhRvD, 70, 043537

Hodge, P.' W. 1961, AJ, 66, 83

Inoue, S. 2011, MNRAS, 416, 1181

Kharchenko, N. V., Piskunov, A. E., Röser, S., Schilbach, E., \& Scholz, R.-D. 2005, A\&A, 438, 1163

King, I. R. 1966, AJ, 71, 64

Klypin, A., Kravtsov, A. V., Valenzuela, O., \& Prada, F. 1999 ApJ, 522, 82

Koposov, S. E., Belokurov, V., Torrealba, G., \& Evans, N. W. 2015a, ApJ, 805, 130

Koposov, S. E., Casey, A. R., Belokurov, V., et al. 2015b, ApJ, 811,62

Lacey, C. G., \& Ostriker, J. P. 1985, ApJ, 299, 633

Laevens, B. P. M., Martin, N. F., Bernard, E. J., et al. 2015, ApJ, 813,44

Lovell, M. R., Eke, V., Frenk, C. S., et al. 2012, MNRAS, 420, 2318

Mackey, A. D., \& Gilmore, G. F. 2003, MNRAS, 340, 175

Maraston, C. 2005, MNRAS, 362, 799 
Martin, N. F., de Jong, J. T. A., \& Rix, H.-W. 2008, ApJ, 684, 1075

Martin, N. F., Ibata, R. A., Chapman, S. C., Irwin, M., \& Lewis, G. F. 2007, MNRAS, 380, 281

McConnachie, A. W. 2012, AJ, 144, 4

Meylan, G., \& Heggie, D. C. 1997, A\&A Rev., 8, 1

Moore, B., Ghigna, S., Governato, F., et al. 1999, ApJ, 524, L19

Muñoz, J. B., Kovetz, E. D., Dai, L., \& Kamionkowski, M. 2016, ArXiv e-prints, 1605.00008

Novak, G. S., Jonsson, P., Primack, J. R., Cox, T. J., \& Dekel, A. 2012, MNRAS, 424, 635

Paczynski, B. 1986, ApJ, 304, 1

Planck Collaboration, Ade, P. A. R., Aghanim, N., et al. 2015 , ArXiv e-prints, 1502.01589

Quinn, D. P., Wilkinson, M. I., Irwin, M. J., et al. 2009, MNRAS, 396, L11
Ricotti, M., Ostriker, J. P., \& Mack, K. J. 2008, ApJ, 680, 829 Sánchez-Salcedo, F. J., Reyes-Iturbide, J., \& Hernandez, X. 2006, MNRAS, 370, 1829

Simon, J. D., \& Geha, M. 2007, ApJ, 670, 313

Simon, J. D., Geha, M., Minor, Q. E., et al. 2011, ApJ, 733, 46 Spergel, D. N., Verde, L., Peiris, H. V., et al. 2003, ApJS, 148, 175 Spitzer, L., Jr. 1969, ApJ, 158, L139

Tisserand, P., Le Guillou, L., Afonso, C., et al. 2007, A\&A, 469, 387

Tremaine, S. D. 1976, ApJ, 203, 72

Yoo, J., Chanamé, J., \& Gould, A. 2004, ApJ, 601, 311 